\title{
Hollow Core Photonic Bandgap Fibers for Mid-IR Applications
}

\author{
N. V. Wheeler, M. N. Petrovich, A. M. Heidt, N. K. Baddela, E. N. Fokoua, J. R. Hayes, S. R. Sandoghchi, \\ F. Poletti and D. J. Richardson \\ Optoelectronics Research Centre, University of Southampton, Highfield, Southampton, SO17 1BJ, UK \\ e-mail: nvw1v10@orc.soton.ac.uk
}

\begin{abstract}
We review our fabrication of low loss $(50 \mathrm{~dB} / \mathrm{km}$ at $3.3 \mu \mathrm{m})$ and low bend sensitivity HC-PBGFs for mid-IR operation. Gas sensing applications are highlighted by a high resolution methane spectrum recorded in $1.26 \mathrm{~m}$ of gas-filled fiber.

OCIS codes: (060.5295) Photonic crystal fibers; (060.2280) Fiber design and fabrication.
\end{abstract}

\section{Introduction}

Hollow core-photonic crystal fibers (HC-PCFs) consist of an air core surrounded by a periodic array of holes, most commonly defined by a silica lattice. The exceptional qualities of HC-PCFs, such as low transmission loss, low bend loss and ultimate low latency and nonlinearity lend these fibers to a wide range of applications. In particular, the extremely low overlap of the fundamental mode with the silica cladding opens up applications in high power beam delivery and gas spectroscopy along with the potential to design these fibers to operate in spectral regions where the material attenuation of silica is high.

One such region is the mid-infrared (IR) which has been receiving increasing interest due to the latest advances in optical sources and detectors for this spectral region. Several all-solid fibers have been successfully fabricated which operate in the mid-IR (e.g. from ZBLAN or chalcogenide glasses [1]), yet these fibers do not possess the outstanding qualities which silica fibers can offer in the near-IR. Recently, anti-resonant (AR) silica-based HC-PCFs have been reported with remarkably low attenuation (e.g. $34 \mathrm{~dB} / \mathrm{km}$ at $3.05 \mu \mathrm{m}$ [2]), reviving interest in fabrication of silica-based HC-PCFs for the mid-IR. However, a current limitation of AR HC-PCFs is bend sensitivity; the fiber reported in [2] exhibits a bend loss of $\sim 6 \mathrm{~dB}$ for a $20 \mathrm{~cm}$ diameter coil.

In this paper we review our recent progress in fabrication of hollow core-photonic bandgap fibers (HC-PBGFs), an alternative form of HC-PCF, for operation in the mid-IR [3]. We report fibers with an attenuation of $50 \mathrm{~dB} / \mathrm{km}$ at $3.3 \mu \mathrm{m}$ (an order of magnitude lower than that previously reported for HC-PBGF designed for the mid-IR) which also exhibit low bend sensitivity and are therefore highly suitable for applications which require a compact device footprint or remote access to difficult areas, such as gas sensing and surgery.

\section{Fiber Fabrication and Characterization}

The HC-PBGFs have a 19 cell core design and 6.5 cladding rings and for fabrication the conventional two-step stack and draw process was used. Optimization of the differential pressure between the core and the cladding regions during the fiber draw, in combination with a thin-core wall surround in the fiber design, enabled loss reduction as well as extension of the usable operating bandwidth. Fig. 1a shows a scanning electron microscope of an example fiber; the average core diameter is $50 \mu \mathrm{m}$, the average hole-to-hole distance is $9.3 \mu \mathrm{m}$ and the relative hole size is

(a)

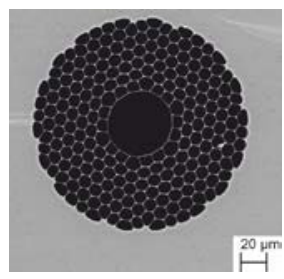

(b)

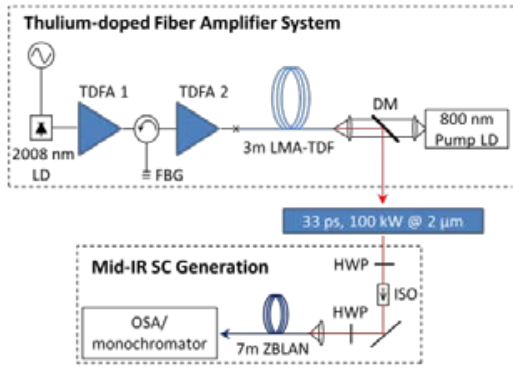

(c)

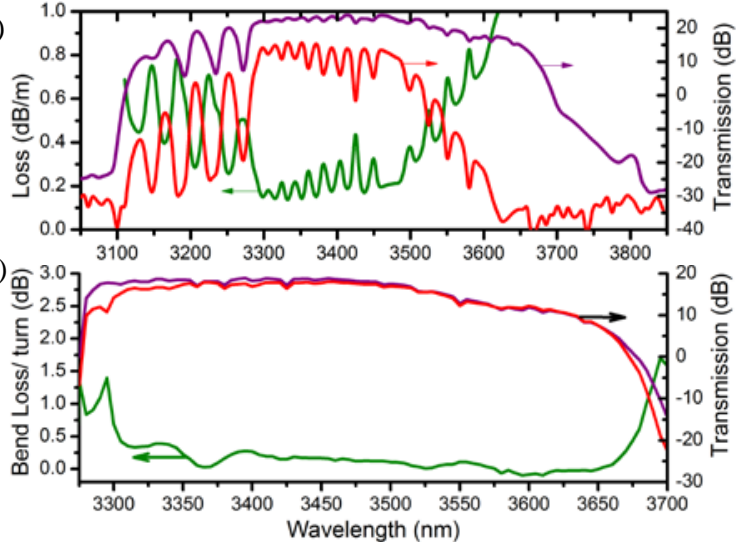

Fig. 1: (a) Scanning electron microscope image of fabricated HC-PBGF, (b) Schematic of custom-built SC source, (c) Cutback loss measurement (green line), from 58m (red line) to $5 \mathrm{~m}$ (purple line), (d) Bend loss measurement (green line) found from comparing loosely coiled fiber (purple line) with the same fiber with 5 times $5 \mathrm{~cm}$ diameter coils. 
0.965. To measure the optical characteristics of the fibers, a custom-built supercontinuum (SC) source was used [4] which extends from 0.75 to $4 \mu \mathrm{m}$ by using a diode seeded picosecond thulium-doped fiber amplifier operating at 2 $\mu \mathrm{m}$ to pump a ZBLAN fiber (Fig. 1b). For detection, a $5 \mathrm{~nm}$ resolution monochromator was initially used. The cutback measurements (Fig. 1c) show that at $\sim 3.3 \mu \mathrm{m}$ a minimum attenuation of $130 \mathrm{~dB} / \mathrm{km}$ is achieved and furthermore, the fiber provides sub $\mathrm{dB} / \mathrm{m}$ guidance for a bandwidth $>500 \mathrm{~nm}$. This fiber therefore provides loss reduction of an order of magnitude compared to previously reported mid-IR HC-PBGFs as well as guidance which extends further into the mid-IR than ever before. Fig. 1d shows that these transmission properties are combined with low bend loss; the bend loss per $5 \mathrm{~cm}$ turn was found to be $<0.25 \mathrm{~dB}$ for a $300 \mathrm{~nm}$ bandwidth.

\section{Gas Spectroscopy in HC-PBGFs}

Fig. $1 \mathrm{~b}$ shows that in the spectral region between 3.3 and $3.6 \mu \mathrm{m}$ several attenuation peaks are recorded in the fiber transmission. These peaks can be attributed to absorption from $\mathrm{HCl}$ gas which is present in the fiber due to the chlorine which is used to dehydrate the bulk silica which is used as the starting material. In Fig. 1b these peaks are not fully resolved, however, due to the high power provided by the SC source it was possible to resolve these lines using a high resolution $(0.2 \mathrm{~nm})$ optical spectrum analyzer for detection. Further cutback measurements using this configuration are shown in Fig. 2a, before and after purging the fiber with Argon gas to remove the HCl. These measurements show that post purging, the measured fiber loss is reduced to $50 \mathrm{~dB} / \mathrm{km}$.

(a)

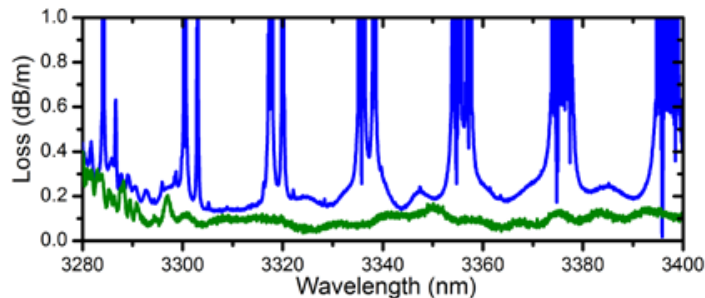

(b)

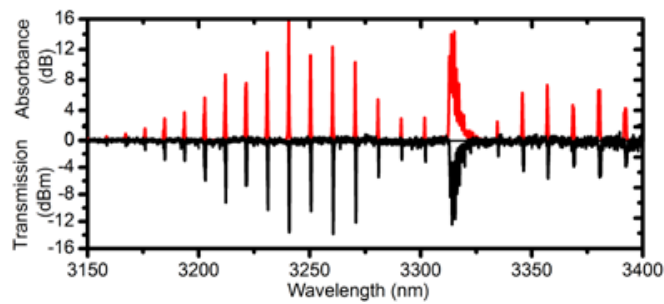

Fig. 2: (a) Cutback loss measurements before and after purging fiber with Argon gas (blue and green lines respectively), (b) Methane spectrum (1000ppm in $\mathrm{N}_{2}$ ) recorded in $1.26 \mathrm{~m}$ of filled HC-PBGF. Experimental data is shown in lower half (black line) and theoretical HITRAN data is shown in top half (red line). All experimental data in figure recorded using $0.2 \mathrm{~nm}$ resolution optical spectrum analyser.

The strong $\mathrm{HCl}$ absorption observed in our fibers highlights the unprecedented interaction length between the guided optical modes and the confined gas which is offered by gas-filled HC-PBGFs. This is particularly of interest in the mid-IR as the fundamental absorption bands of gas species key to applications such as environmental and industrial sensing lie in this spectral region. These absorption bands are orders of magnitude stronger than their near-IR overtones and therefore provide the means to significantly increase the sensitivity achievable in gas sensing

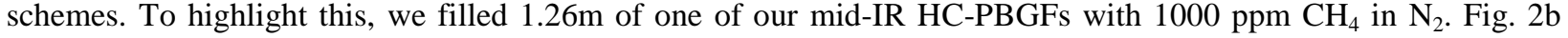
shows the recorded transmission spectrum (lower half) in excellent agreement with theoretical data from HITRAN [5] (top half). We envisage that with use of longer fibers, in combination with more sophisticated detection techniques, sensitivity levels of the order of ppb will be easily achievable in these fibers.

\section{Conclusions}

We have reported our recent progress in fabrication of low loss and low bend sensitivity HC-PBGFs for the mid-IR and highlighted their suitability for exploitation of fundamental gas absorption bands for use in gas sensing devices. We anticipate that further loss reduction, as well as the means to push the guidance band deeper into the mid-IR, can be achieved by enlarging the core defect to reduce the overlap of the fundamental mode with the silica cladding. This work was supported by the UK EPSRC (EP/H02607X/1). A.M.H. acknowledges funding from EU FP7 Marie Curie Actions (300859 ADMIRATION). F.P. and D.J.R. gratefully acknowledge support from the Royal Society.

\section{References}

[1] J. A. Harrington, Infrared Fibers and Their Applications. Bellingham, Washington USA: Society of Photo-Optical Instrumentation Engineers ., 2004.

[2] F. Yu, W. J. Wadsworth, and J. C. Knight, "Low loss silica hollow core fibers for 3-4 $\mu \mathrm{m}$ spectral region.," Opt. Express, vol. 20 , no. 10, pp. 11153-8, May 2012.

[3] N. V Wheeler et al. "Low-loss and low-bend-sensitivity mid-infrared guidance in a hollow-core-photonic-bandgap fiber.," Opt. Lett., vol. 39, no. 2, pp. 295-8, Jan. 2014.

[4] A. M. Heidt et al. "Mid-infrared ZBLAN fiber supercontinuum source using picosecond diode-pumping at $2 \mu \mathrm{m}$," Opt. Express, vol. 21, no. 20, p. 24281, Oct. 2013.

[5] L. S. Rothman et al. “The HITRAN 2008 molecular spectroscopic database,” J. Quant. Spectrosc. Radiat. Transf., vol. 110, no. 9-10, pp. 533-572, 2009. 